\title{
La Formación de los profesionales sanitarios en prevención de riesgos laborales
}

\section{Training of health professionals in occupational risk prevention}

\author{
Ascensión Bernal Zamora \\ Subdirección General de Servicios Aplicados Formación e Investigación. \\ Instituto de Salud Carlos III. Madrid. España.
}

\author{
Jerónimo Maqueda Blasco \\ Escuela Nacional de Medicina del Trabajo. Instituto de Salud Carlos III. \\ Madrid. España.

\section{Correspondencia:} \\ Jerónimo Maqueda Blasco \\ Escuela Nacional de Medicina del Trabajo \\ Pabellón 8. Ciudad Universitaria \\ 28040 Madrid. España \\ Tfno: 918224011 \\ Fax: 915447073 \\ e-mail: jmaqueda@isciii.es
}

La progresiva mejora de nuestro sistema preventivo ha sido un esfuerzo constante de las Administraciones Públicas por lograr entornos de trabajo seguros y saludables que permitan mejorar los indicadores de salud de nuestra población trabajadora y por lo tanto su productividad y calidad de vida.

La formación constituye un desarrollo necesario para el fortalecimiento de los recursos humanos en prevención de riesgos laborales a través de la mejora de la competencia, aspecto que tiene una mayor relevancia cuando hablamos de los profesionales sanitarios en prevención de riesgos laborales dado que su intervención es la persona.

El avance de estos últimos años en la formación de los profesionales sanitarios tanto de Medicina como de enfermería del trabajo han sido claros.

El nivel de exigencia de los actuales programas de especialización vía MIR (Médicos Internos Residentes) y vía EIR (Enfermeros Internos Residentes) marcan las bases para una práctica profesional de mayor calidad, al mismo tiempo que supone un reconocimiento de la relevancia del papel social y sanitario de estas especialidades en el conjunto de las profesiones sanitarias.

El Instituto de Salud Carlos III ha sido solidario con el esfuerzo realizado por las distintas Comunidades Autónomas en el desarrollo de la ORDEN SCO/1526/2005, de 5 de mayo, por la que se aprueba y publica el programa formativo de la especialidad de Medicina del Trabajo.

La progresiva extensión de este programa permite que en la actualidad la Medicina del Trabajo sea una especialidad presente en la oferta de formación especializada en 16 Comunidades Autónomas, 11 de las cuales comparten este proyecto con la Escuela Nacional de Medicina del Trabajo, a cuyos Jefes de Estudios traslado el reconocimiento por su trabajo y el agradecimiento por su confianza y por sus contribuciones a la mejora de los procedimientos académicos y docentes que en materia MIR imparte la Escuela Nacional de Medicina del Trabajo.

Este reconocimiento debo ampliarlo a la Subdirección General de Ordenación Profesional del Ministerio de Sanidad y Política Social que como autoridad en materia de formación sanitaria trabaja por la mejora de nuestro sistema de especialización. 
La formación MIR en Medicina del Trabajo está afrontando el nuevo reto de la reforma del sistema de especialización. El nuevo sistema de troncalidad favorecerá una formación interdisciplinar lo que supondrá un mutuo conocimiento y entendimiento entre especialidades, pero al mismo tiempo supone re-diseñar itinerarios formativos, aspecto relevante en el caso de la Medicina del Trabajo, por una doble razón: La falta de formación sobre esta disciplina en el pre-grado y el ámbito específico de su ejercicio, enmarcado por la normativa laboral y de Seguridad Social.

Esta migración del programa de formación del MIR de Medicina del Trabajo al nuevo sistema de troncalidad, debe valorar especialmente esta circunstancia y en ese sentido ponemos a disposición de la Subdirección General de Ordenación Profesional, la experiencia que hemos adquirido durante estos cinco años de vigencia del actual programa formativo que nos ha permitido identificar las debilidades con las que inician los residentes su periodo de especialización y que, por lo tanto, necesitan un refuerzo en el nuevo sistema de troncalidad.

La especialización en Enfermería del Trabajo, debe ser también un tema de especial interés para el conjunto de instituciones públicas y asociaciones profesionales, implicadas en la prevención de riesgo laborales.

El REAL DECRETO 450/2005, de 22 de abril, sobre especialidades de Enfermería, da un importante impulso a la formación en enfermería del trabajo, trasladándola desde la Diplomatura de Enfermería del empresa al espacio EIR de formación, incorporándose a las Comisiones Docentes Multiprofesionales.

A pesar de que su programa formativo se aprobara en 2009 a través de la Orden SAS/1348/2009, la oferta de formación que se ha ofrecido en el conjunto del Estado ha ido muy por debajo de la importancia que esta especialidad tiene para nuestro sistema preventivo. Las trece plazas ofertadas en la convocatoria EIR 2010-2011, son claramente insuficientes para cubrir el espacio propio que esta especialidad tiene en el desarrollo de actuaciones preventivas.

Ambas especialidades comparten el problema de la financiación, que no deja de ser reflejo de una dualidad. Mientras que el sistema de formación anida en el Sistema Nacional de Salud, el Espacio profesional radica o bien en el Sistema de Seguridad Social o bien en el Sector Privado. El debate sobre la financiación de la formación de los profesionales sanitarios de prevención de riesgos laborales, reclamado por interlocutores sociales, sociedades científicas y en su conjunto por la Sociedad del Trabajo, constituye hoy en día una necesidad no resuelta.

Sostener un alto nivel de competencia y adecuar éstas a las necesidades que emergen en el tejido productivo es parte del trabajo de la Instituciones que estamos comprometidas con los avances en la calidad de vida en el trabajo.

Garantizar un formación continua: actual, accesible y acreditada, constituye también un área de desarrollo de especial interés para el ISCIII, en una doble proyección:

- Garantizar la sostenibilidad de la competencia del profesional sanitario de prevención en ejercicio.

- Generar unos conocimientos en torno al trabajo como determinante de la salud en otras profesiones sanitarias.

La oferta de formación dirigida desde las Administraciones Públicas debe

- Responder a las necesidades del profesional en ejercicio.

- Innovar y desarrollar nuevos proyectos y figuras curriculares como las previstas por la Ley de Ordenación de las Profesiones Sanitarias (Ley 44/2003), como la Capacitación Específica y los Diplomas de Acreditación Avanzada.

- Favorecer la accesibilidad a la formación mediante el incremento de la oferta formativa en la modalidad de e-aprendizaje.

Si bien la formación continua de los profesionales sanitarios tiene un desarrollo normativo propio, claramente diferenciado de la formación de post- grado de otras profesiones, es indudable el valor añadido de establecer una cooperación con la Universidad que facilite el desarrollo de proyectos curriculares comunes en líneas de títulos propios o masteres oficiales.

La formación de los profesionales sanitarios de prevención de riesgos laborales no debe limitarse a la mera trasmisión de conocimientos sino que debe introducir valores y compromisos con el desarrollo científico 
de las propias especialidades y disciplinas. Promover la investigación desde los programas docentes es un objetivo académico ineludible. Se trata de comprometer al especialista en formación en el proceso de generar conocimiento, la investigación no sólo es la única estrategia posible para el desarrollo, la innovación y el diseño de políticas de anticipación, sino que es necesaria para una mejora de la práctica clínica y preventiva tanto en medicina del trabajo como en valoración médica. Una practica basada en la evidencia científica da a nuestras decisiones mayor eficacia, eficiencia y equidad.

Confiando que avancemos en estos objetivos durante este año declaro inaugurado el año académico 2010- 2011 de la Escuela Nacional de Medicina del Trabajo del Instituto de Salud Carlos III. 